\title{
Histological changes caused by Chronic Sleep Deprivation on Rats Temporomandibular Joints
}

\author{
Original \\ Article \\ Khaled Ibrahim Barakat, \\ Ahmed \\ Sayed Hassan \\ Khidr \\ and \\ Hamed Mohamed Ahmed Gad \\ Department of Oral and Maxillofacial Surgery, Faculty of Dentistry, Minia \\ University, Minia, Egypt
}

\begin{abstract}
Introduction: The temporomandibular joint (TMJ) known as ginglymoarthrodial joint is a complex synovial system composed of two joints together with their articulating ligaments and masticatory muscles.

Objectives: To assess the histological effects of chronic sleep deprivation (CSD) on the temporomandibular joint (TMJ) (Experimental study).

Methods: The study was conducted on16 Albino rats, aged from $(8-10)$ weeks, and weighed from 190 to 210 gm. Rats were randomly allocated into two equal groups, a chronic sleep deprivation group (CSD), and a control group (CTRL). Each group was housed in a separate home cage but in the same room with free access to food and water. Rats of CSD group were subjected to CSD using the modified multiple platform method (MMPM) 18 hours per day for 3 weeks. Histomorphology of TMJ articulating surfaces were observed using light microscope (LM).

Results: LM showed significant pathological alterations in the TMJ in CSD group. Conclusion: CSD causes pathological changes in the articulating surfaces of TMJ.
\end{abstract}

Key Words: Chronic Sleep Deprivation, Temporomandibular Joints.

Received: 18 February 2020, Accepted: 13 March 2020.

Corresponding Author: Khaled Ibrahim Barakat, Department of Oral and Maxillofacial Surgery, Faculty of Dentistry, Minia University, Minia, Egypt, Tel.: +20222677077, Mobile: +201222324596, E-mail: send2kbarakat@yahoo.com.

ISSN: 2090-097X, July 2019, Vol. 10, No. 3

\section{INTRODUCTION}

The temporomandibular joint (TMJ) also known as ginglymoarthrodial joint is a complex synovial system composed of two joints together with their articulating ligaments and masticatory muscles ${ }^{[1]}$.

Temporomandibular disorders (TMDs) is a collective term that includes a number of clinical complaints involving the muscles of mastication, the TMJ, or associated orofacial structures ${ }^{[2]}$. These disorders are highly prevalent, with variable percentages among different populations. TMD was previously defined as "functional disturbances of the masticatory system" [3], others included masticatory muscle disorders, degenerative and inflammatory TMJ disorders ${ }^{[4]}$, and TMJ disc displacements ${ }^{[5]}$ under the definition of TMD. TMDs have serious effects regarding patient's daily life and his activity at various levels including; physical incapability, impaired sleep quality and reduced learning abilities as well as its impact on expenditures ${ }^{[6]}$.

The causes of TMDs are complex and multifactorial ${ }^{[1]}$. Factors affecting TMDs were classified into; Predisposing factors, Initiating factors and Perpetuating factors ${ }^{[7]}$. The perpetuating factors include: Behavioral factors, social factors, emotional factors such as depression and anxiety and cognitive factors such as negative thoughts and attitudes which can make resolution of the illness more difficult ${ }^{[1]}$.
Among various contributing factors, the psychological factors involved in the etiology of TMD have remained controversial until Schwartz realized that TMD and psychological factors are closely related ${ }^{[8,9]}$. This relation had attracted the attention of many researchers and they started to investigate the role of stress in aggravating TMD signs and symptoms ${ }^{[10]}$. Consequently it was suggested that psychological factors are closely related to the occurrence, development, and effective treatment of $\mathrm{TMD}^{[11]}$. Therefore, the treatment of TMDs had been transformed recently from a biomedical model into a biopsychosocial mode ${ }^{[12]}$, with increasing numbers of researches adopted that TMD is a psychosomatic disease ${ }^{[13]}$.

The complexity of factors affecting TMDs, and its variable subtypes resulted in lack of reliable accurate standardized methodology for diagnosis and evaluation of the clinical outcomes of variable treatment modalities in TMDs researches ${ }^{[14]}$. This led to the development of The Research Diagnostic Criteria for Temporomandibular Disorders (RDC/TMD) which provide clinical researchers with a standardized system that can be evaluated for its use in examining, diagnosing, and classifying the most common subtypes of $\mathrm{TMDs}^{[15,16]}$. One of the major attributes of the RDC/TMD that make them especially valuable in clinical research settings is use of a dual-axis system: Axis I to record clinical physical findings, and Axis II to record behavioral, psychological and psychosocial 
status $^{[17]}$. RDC/TMD was widely accepted and applied, and it was later revised into DC/TMD to reach a more complete and specific model ${ }^{[18]}$.

Although the psychological factors are considered among the etiological factors of TMDs, only a few studies have focused on whether psychological factors can lead to pathological changes in the TMJ or not ${ }^{[19,20]}$. Sleep is an important factor associated with $\mathrm{TMD}^{[21]}$. Studies have consistently shown that the majority of patients with TMD report poor sleep quality and the subjective ratings of poor sleep are associated with increased severity of clinical pain and psychological distress Histologically TMD can be reflected as pathological changes in the articular surfaces, articulating disc and capsule ${ }^{[22]}$

Xiaowen et al. ${ }^{[23]}$, in their study on rats in 2016 demonstrated that chronic sleep deprivation (CSD) markedly affected bone mass and bone metabolism, by lowering bone mineral density (BMD), deteriorating bone microarchitecture, and decreasing bone formation and resorption markers. Another study by $\mathrm{Ma} \mathrm{C}$ et al. ${ }^{[20]}$ concluded that CSD causes pathological changes in the TMJ. Other studies conducted on rats concluded that CSD increased pain related factors ${ }^{[24]}$, affected the normal bone metabolism $^{[25]}$ and caused osteoarthritic-like pathological alterations in the rat $\mathrm{TMJ}^{[26]}$. The study of Liang $\mathrm{C}$ et al. ${ }^{[27]}$ in 2019 confirmed these results. Since rats can provide a good histological model relative to human TMJ we used experimental rats model to investigate the effect of sleep deprivation $^{[28]}$. Thus the purpose of the current study is to confirm the expected inflammatory and pathologic changes caused by CSD on the articulating surfaces and synovial tissues of TMJ.

\section{MATERIALS AND METHODS}

The study was conducted on 16 Albino rats, aged from (8-10) weeks, and weighed from 190 to $210 \mathrm{gm}$. Rats were randomly allocated into two equal groups, a chronic sleep deprivation group (CSD), and a control group (CTRL).
Each group was housed in a separate home cage but in the same room with free access to food and water.

For sleep CSD group we used the Modified Multiple Platform Method (MMPM) as described by Suchecki and Tufik ${ }^{[29]}$ with minimal modification (Figure 1). We assembled two glass boxes of dimensions $(115 \mathrm{~cm}$ length $\mathrm{X}$ $75 \mathrm{~cm}$ width $X 120 \mathrm{~cm}$ height). Fifteen narrow cylindrical glass platforms $(6.5 \mathrm{~cm}$ in diameter $)$ were attached to the floor of each box with $15 \mathrm{~cm}$ distance between each other. An additional stainless-steel grid floor covering was placed above the narrow circular platforms in the box of the control group. The two boxes were filled with water up to $1 \mathrm{~cm}$ of the upper surfaces of the platforms. In CSD box, the rats were placed on the platforms. In these conditions, the rats could move around freely inside the box by jumping from one platform to another. When they reached the paradoxical phase of sleep, their faces touched the water; which subsequently woke them up. On the other hand, rats of control group were allowed to lie down on the metal grid without falling into the water, while their tails may still touch the water.

The rats were placed in the boxes for 15 minutes per day for 5 days before the beginning of the experiment for accommodation to the experimental conditions. Then animals were subjected to the experimental conditions for 18 hours per day; from $2 \mathrm{pm}$ to $8 \mathrm{am}$, and were allowed to sleep freely in their home boxes for the remaining 6 hours of the day. The experiment was continued for 3 weeks through which the rats were subjected to CSD conditions. Temperature was monitored to be $23+/-2^{\circ} \mathrm{C}$. Food and water were offered freely to the animals. The water of the tanks was changed daily throughout the time of the experiment to provide a constant water level and maintain proper hygienic conditions. After the 30 days period of the experiment, rats were euthanized by cervical dislocation ${ }^{[30]}$, and TMJs were harvested. Specimens were prepared to be examined under light microscope (LM) using hematoxylin and eosin stains.

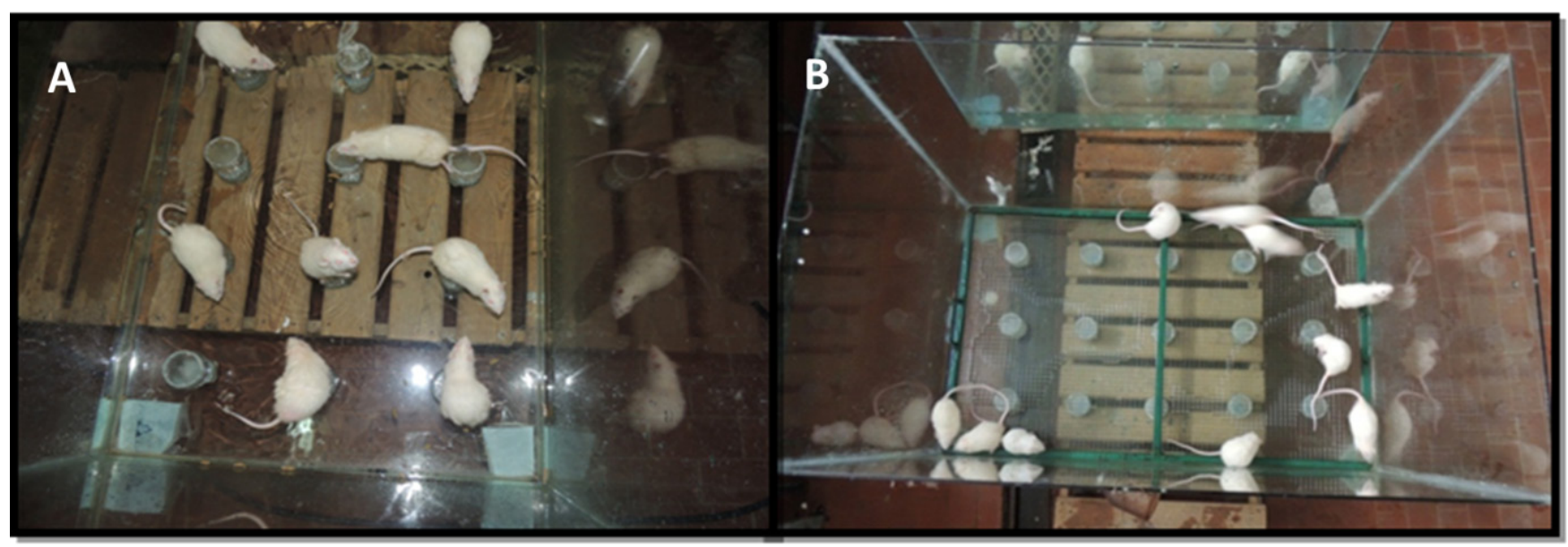

Figure 1: CSD Model; A: animals of CSD group. B: animals of CTRL group. 


\section{Specimen preparation for light microscope:}

Specimens were fixed in neutral buffer formalin $10 \%$, washed in (PBS), decalcified in formic acid. The samples were trimmed and washed again in PBS, dehydrated in ascending grades of ethanol, cleared in zylene, infiltrated and embedded in paraffin wax. Serial sections of 5 microns were obtained in sagittal plane and stained with Hematoxylin and Eosin ${ }^{[31]}$. Samples were examined using Olympus ${ }^{\circledR}$ CX31 LM.

\section{RESULTS}

Some behavioral and featural changes were noticed on the rats of CSD group, such as general weakness, delayed responses, hypomobility, loss of weight, bulging of the eyes and the fur became ragged and puffed up. The frequency of the rats seen falling into the water increased as the experiment proceeded.

\section{Histological observations:}

The condyle of the CTR group displayed characteristic zonal cellular arrangements with distinct regions in the articular cartilage. The TMJ appeared normal, with a smooth condylar cartilage surface, and chondrocytes were normally distributed throughout the cartilage. In case of CSD group, the fibrous articular surfaces of the condylar cartilages became visibly tougher, and a debonding of fibrous layer was observed. Distorted collagen fibers were also observed (Figure 2).

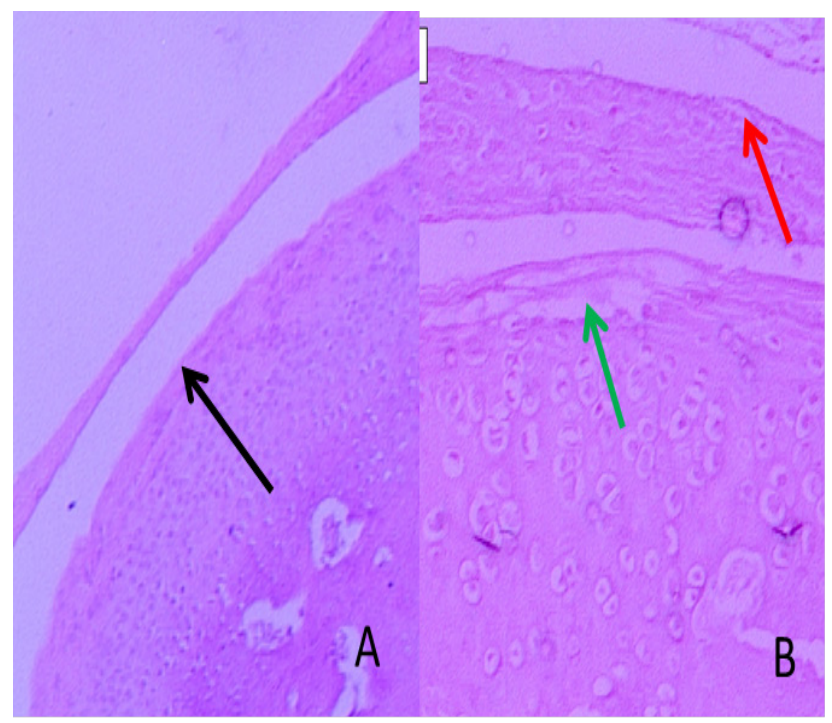

Figure 2: Sagittal section of TMJ stained with $\mathrm{H}$ and $\mathrm{E}$, condyle and articulating disc under LM. A: CTRL group. Black arrow: smooth condylar surface. B: CSD group. Red arrow: disc irregularities. Green arrow: distorted condylar surface.

\section{DISCUSSION}

Recently, the treatment of temporomandibular disorders (TMDs) has been transformed from a biomedical model into a biopsychosocial model. The role of psychological factors in TMD pathogenesis has received increasing attention $^{[12,}{ }^{32]}$. Many studies have confirmed that psychological factors such as sleep disorders and psychological stress are strongly related to TMJ

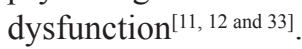

In the present study we established the animal model of CSD by the modified multiple platform method. The MMPM induces paradoxical sleep deprivation to rats without any additional stress, such as social isolation or instability. Using this effective animal model of CSD, we were able to observe the direct effect of experimentally induced SD on TMJ pathology.

Generally, psychological stress has been accompanied by regional modification of the $\mathrm{TMJ}^{[34]}$. In this study, we observed clear changes in the histopathology of the rat TMJ after CSD. The histopathological findings were mainly in the condyle and articular disc and included tough fibrous articular surfaces, distorted collagen fibers and debonding fibrous layer. Our results proved that psychological stress alone can induce pathological changes in the rats TMJ.

The present study has some limitations regarding the relatively small sample size and the short duration of the experiment. Moreover, the absence of other means of evaluation to support the results, such as evaluation of the expression of the inflammatory mediators in the joint synovial tissues to confirm the presence of actual inflammatory process, and electron microscopy to evaluate the changes in the ultrastructure of the articulating surfaces of the joint.

\section{DISCUSSION}

Chronic sleep deprivation induces alteration in the histomorphology of TMJ articulating surfaces. These findings provide evidence for the possible involvement of sleep disturbances in the onset and progression of TMD, indicating that such parameters should be taken into consideration in the management of TMJ disorders.

\section{CONFLICT OF INTEREST}

The authors declare no conflict of interest.

\section{REFERENCES}

1. Sharma S, Gupta DS, Pal US, Jurel SK. Etiological factors of temporomandibular joint disorders. Natl J Maxillofac Surg. 2011; 2(2): 116 - 9.

2. Okeson JP, de Leeuw R. Differential Diagnosis of Temporomandibular Disorders and Other Orofacial Pain Disorders. Dental Clinics of North America. 2011; 55(1): 105 - 20. 
3. Agerberg G, Carlsson GE. Symptoms of functional disturbances of the masticatory system. A comparison of frequencies in a population sample and in a group of patients. Acta Odontol Scand. 1975; 33(4): 183 - 90.

4. Guideline on Acquired Temporomandibular Disorders in Infants, Children, and Adolescents. Pediatr Dent. 2016; 38(6): 308 - 14.

5. Murphy MK, MacBarb RF, Wong ME, Athanasiou KA. Temporomandibular disorders: a review of etiology, clinical management, and tissue engineering strategies. Int $\mathrm{J}$ Oral Maxillofac Implants. 2013; 28(6): e393 - 414.

6. Palermo TM. Impact of recurrent and chronic pain on child and family daily functioning: a critical review of the literature. J Dev Behav Pediatr. 2000; 21(1): 58 - 69 .

7. Suvinen TI, Reade PC, Kemppainen P, Kononen M, Dworkin SF. Review of aetiological concepts of temporomandibular pain disorders: towards a biopsychosocial model for integration of physical disorder factors with psychological and psychosocial illness impact factors. Eur J Pain. 2005; 9(6): 613 - 33 .

8. Schwartz LL. Pain associated with the temporomandibular joint. The Journal of the American Dental Association. 1955; 51(4): 394 - 7.

9. Weinberg LA. The role of stress, occlusion, and condyle position in TMJ dysfunction-pain. The Journal of prosthetic dentistry. 1983; 49(4): 532 - 45.

10. Kanehira H, Agariguchi A, Kato H, Yoshimine $\mathrm{S}$, Inoue $\mathrm{H}$. Association between stress and temporomandibular disorder. Nihon Hotetsu Shika Gakkai Zasshi. 2008; 52(3): 375 - 80.

11. Macfarlane TV, Kenealy $\mathrm{P}$, Anne Kingdon $\mathrm{H}$, Mohlin B, Pilley JR, Mwangi CW, et al. Orofacial pain in young adults and associated childhood and adulthood factors: results of the population study, Wales, United Kingdom. Community dentistry and oral epidemiology. 2009; 37(5): 438 - 50.

12. Kafas $\mathrm{P}$, Leeson R. Assessment of pain in temporomandibular disorders: the bio- psychosocial complexity. International Journal of Oral and Maxillofacial Surgery. 2006; 35(2): 145 - 9.

13. Wu G, Chen L, Wei G, Li Y, Zhu G, Zhao Z, et al. Effects of sleep deprivation on pain-related factors in the temporomandibular joint. J Surg Res. 2014; 192(1): 103 - 11.

14. Undt G, Murakami K-I, Rasse M, Ewers R. Open versus arthroscopic surgery for internal derangement of the temporomandibular joint: A retrospective study comparing two centres' results using the Jaw Pain and Function Questionnaire. Journal of Cranio-Maxillofacial Surgery. 2006; 34(4): 234 - 41.

15. Dworkin SF. Research diagnostic criteria for temporomandibular disorders: review, criteria, examinations and specifications, critique. J craniomandib Disord. 1992; 6: 301 - 55.

16. Barakat K. Correlation between pain and arthroscopic features of synovitis: A logical approach to verify correlations of TMJ pain. Tanta Dental Journal. 2013; 10(3): 168 - 72.

17. Dworkin SF, Sherman J, Mancl L, Ohrbach R, LeResche L, Truelove E. Reliability, validity, and clinical utility of the research diagnostic criteria for Temporomandibular Disorders Axis II Scales: depression, non-specific physical symptoms, and graded chronic pain. Journal of orofacial pain. 2002; 16(3): 207 - 20.

18. Schiffman E, Ohrbach R, Truelove E, Look $\mathrm{J}$, Anderson G, Goulet J-P, et al. Diagnostic criteria for temporomandibular disorders (DC/ TMD) for clinical and research applications: recommendations of the International RDC/TMD Consortium Network and Orofacial Pain Special Interest Group. Journal of oral \& facial pain and headache. $2014 ; 28(1): 6$.

19. Li Q, Zhang M, Chen YJ, Zhou Q, Wang YJ, Liu J. Psychological stress alters microstructure of the mandibular condyle in rats. Physiology \& behavior. 2013; 11039-129:111-

20. Ma C, Wu G, Wang Z, Wang P, Wu L, Zhu G, et al. Effects of chronic sleep deprivation on the extracellular signal-regulated kinase pathway in the temporomandibular joint of rats. PLoS One. 2014; 9(9): e107544. 
21. Goldberg JI. Association of Sleep Quality and Painful Temporomandibular Disorders: State University of New York at Buffalo; 2013.

22. Yatani H, Studts J, Cordova M, Carlson CR, Okeson JP. Comparison of sleep quality and clinical and psychologic characteristics in patients with temporomandibular disorders. J Orofac Pain. 2002; 16(3): $221-8$.

23. Xu X, Wang L, Chen L, Su T, Zhang Y, Wang T, et al. Effects of chronic sleep deprivation on bone mass and bone metabolism in rats. J Orthop Surg Res. 2016; 11(1): 87.

24. Wu G, Chen L, Wei G, Li Y, Zhu G, Zhao Z, et al. Effects of sleep deprivation on pain-related factors in the temporomandibular joint. Journal of Surgical Research. 2014; 192(1): 103 - 11.

25. Geng W, Wu G, Huang F, Zhu Y, Nie J, He $\mathrm{Y}$, et al. Sleep deprivation induces abnormal bone metabolism in temporomandibular joint. International journal of clinical and experimental medicine. 2015; 8(1): 395.

26. Dong Y, Wu G, Zhu T, Chen H, Zhu Y, Zhu G, et al. VEGF promotes cartilage angiogenesis by phospho-ERK12/ activation of D114 signaling in temporomandibular joint osteoarthritis caused by chronic sleep disturbance in Wistar rats. Oncotarget. 2017; 8(11): 17849.

27. Liang C, Yang T, Wu G, Li J, Geng W. Therapeutic effect of low-intensity pulsed ultrasound on temporomandibular joint injury induced by chronic sleep deprivation in rats. American journal of translational research. 2019; 11(6): 3328.

28. Porto GG, Vasconcelos BCdE, Andrade ESdS, Silva-Junior VA. Comparison between human and rat TMJ: anatomic and histopathologic features. Acta cirurgica brasileira. 2010; 25(3): 290 - 3.

29. Suchecki D, Duarte Palma B, Tufik S. Sleep rebound in animals deprived of paradoxical sleep by the modified multiple platform method. Brain Res. 2000; 875(122-14 :(2-.

30. Leary SL, Underwood W, Anthony R, Cartner S, Corey D, Grandin T, et al. AVMA guidelines for the euthanasia of animals: 2013 edition. 2013: American Veterinary Medical Association Schaumburg, IL; 2013.

31. Bancroft J, Stevens A. Bone and the preparation of bone sections. Theory and Practice of Histological Techniques: Churchill Livingstone, New York; 1982. p. 297.

32. Amin MF, Hassan AM, Barakat KI. The accuracy of dynamic Magnetic Resonance Imaging in evaluation of internal derangement of the temporomandibular joint; comparison with arthroscopic findings. The Egyptian Journal of Radiology and Nuclear Medicine. 2012; 43(3): 429 - 36.

33. Sanders AE, Akinkugbe AA, Bair E, Fillingim RB, Greenspan JD, Ohrbach R, et al. Subjective sleep quality deteriorates before development of painful temporomandibular disorder. The Journal of Pain. 2016; 17(6): 669 - 77.

34. Wu G, Chen L, Zhu G, Su Y, Chen Y, Sun J, et al. Psychological stress induces alterations in temporomandibular joint ultrastructure in a rat model of temporomandibular disorder. Oral Surgery, Oral Medicine, Oral Pathology, Oral Radiology, and Endodontology. 2011; 112(6): e106-e12. 